\title{
A brain natriuretic peptide-based prediction model for atrial fibrillation after thoracic surgery: Development and internal validation
}

\author{
David Amar, MD, ${ }^{\mathrm{a}}$ Hao Zhang, MD, ${ }^{\mathrm{a}}$ Kay See Tan, PhD, ${ }^{\mathrm{b}}$ Daniel Piening, BS, ${ }^{\mathrm{a}}$ Valerie W. Rusch, $\mathrm{MD},{ }^{\mathrm{c}}$ and
} David R. Jones, $\mathrm{MD}^{\mathrm{c}}$

\section{ABSTRACT}

Objective: Postoperative atrial fibrillation (POAF) is common after anatomic thoracic surgery. Elevated preoperative brain natriuretic peptide (BNP) level is strongly associated with risk of POAF. We describe the development and internal validation of a clinical prediction model for POAF that includes BNP and other clinical factors.

Methods: Clinical and preoperative BNP data were collected for 635 patients in sinus rhythm before anatomic lung $(n=540)$ or esophageal $(n=95)$ resection. The primary outcome was new onset of POAF ( $>5$ minutes) during hospitalization. A prediction model was developed using multivariable logistic regression analysis and internally validated using a bootstrap-resampling approach.

Results: POAF occurred in $20 \%$ of patients (124 out of 635). BNP level was higher among patients with than without POAF (median, 45 vs $23 \mathrm{pg} / \mathrm{mL}$; $P<.0001$ ). The final prediction model included 5 factors: age (odds ratio [OR], $1.05 ; 95 \%$ confidence interval $[\mathrm{CI}], 1.02-1.08 ; P=.001)$, body mass index (OR, 1.05; 95\% CI, 1.00-1.09; $P=.016)$, BNP level (75th vs 25th percentile, 57.5 vs $12.5 \mathrm{pg} / \mathrm{mL}$; OR, 2.08; 95\% CI, 1.26-3.43; $P=.0003)$, history of atrial fibrillation (OR, 5.91; 95\% CI, 2.47-14.11; $P<.0001$ ), and extent of surgery (compared with segmentectomy [reference]: pneumonectomy OR, 6.70; 95\% CI, 1.91-24.70; esophagectomy OR, 4.93; 95\% CI, 1.94-14.06; lobectomy OR, 1.88; 95\% CI, 4.90-8.34; overall $P=.0002)$. The model had good calibration and discrimination ( $C$ statistic, 0.736). After internal validation, optimismcorrected measures showed similarly good calibration and discrimination $(C$ statistic, 0.720; 95\% CI, 0.664-0.765).

Conclusions: Our novel prediction model-based interactive calculator can be used to identify patients at high risk of POAF and could be incorporated into practice prevention guidelines. (J Thorac Cardiovasc Surg 2019;157:2493-9)

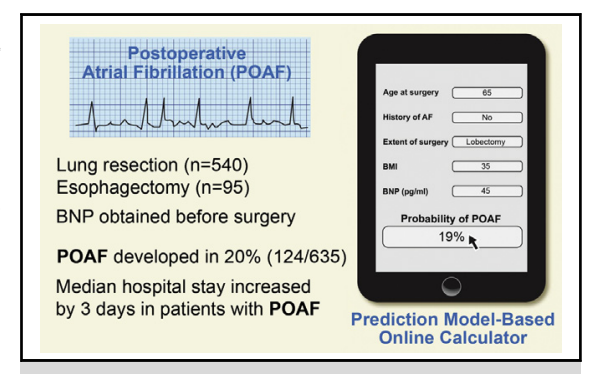

The study results and interactive calculator.

\section{Central Message}

We developed a prediction model and interactive calculator using preoperative clinical variables and brain natriuretic peptide level that predicts probability of postoperative atrial fibrillation.

\section{Perspective}

Postoperative atrial fibrillation is common after noncardiac thoracic surgery and may be associated with longer hospital stay and greater risk of stroke. We developed and internally validated a novel brain natriuretic peptide-based prediction model using easily available preoperative clinical variables and an interactive calculator for probability of postoperative atrial fibrillation.

See Commentaries on pages 2500 and 2502.
From the Departments of ${ }^{\mathrm{a} A n e s t h e s i o l o g y}$ and Critical Care Medicine, ${ }^{\mathrm{b}}$ Epidemiology and Biostatistics, and ${ }^{\mathrm{c}}$ Surgery, Memorial Sloan Kettering Cancer Center, New York, NY.

Supported in part by National Institutes of Health Cancer Center Support grant P30 CA008748.

Received for publication June 13, 2018; revisions received Jan 11, 2019; accepted for publication Jan 21, 2019; available ahead of print Feb 28, 2019.

Address for reprints: David Amar, MD, Department of Anesthesiology and Critical Care Medicine, Memorial Sloan Kettering Cancer Center, 1275 York Ave, M-304, New York, NY 10021 (E-mail: amard@mskcc.org). $0022-5223 / \$ 36.00$

Copyright (C) 2019 by The American Association for Thoracic Surgery https://doi.org/10.1016/j.jtcvs.2019.01.075
Postoperative atrial fibrillation (POAF) occurs in $16 \%$ of patients after noncardiac thoracic surgery and may be associated with prolonged hospital stay and short- and longterm risk of ischemic stroke. ${ }^{1-3}$ Patient age, male gender, and extent of surgical resection are known independent risk factors for POAF. ${ }^{1,2}$ Mechanisms that have been

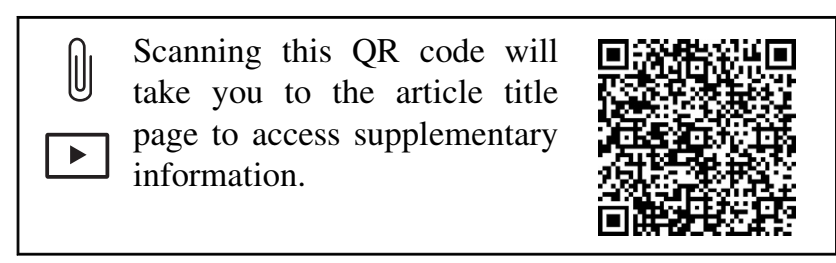




\section{Abbreviations and Acronyms \\ $\mathrm{AF}=$ atrial fibrillation \\ BMI = body mass index \\ $\mathrm{BNP}=$ brain natriuretic peptide \\ $\mathrm{POAF}=$ postoperative atrial fibrillation \\ VATS $=$ video-assisted thoracic surgery}

implicated in predisposing postsurgical patients to reentrant arrhythmias such as POAF include altered sympathovagal balance, operative injury to autonomic hilar nerve fibers, and/or oxidative and inflammatory changes. ${ }^{2,4} \mathrm{We}$ and others have recently shown that preoperative echocardiographic indices of atrial enlargement and diastolic dysfunction are associated with risk of POAF after thoracic surgery. ${ }^{5-7}$ Echocardiography is not performed routinely before thoracic surgery, and, if it is performed, the special measurements needed to detect these factors require significant expertise and additional costs. By comparison, blood biomarkers are easy to analyze. An accumulating body of literature illustrates that increased levels of brain natriuretic peptide (BNP) are associated with postoperative major adverse cardiovascular events in general $^{8,9}$ and POAF in particular. ${ }^{10-12}$ On the basis of our previous work examining the association between BNP and POAF, we sought to develop and internally validate a prediction model for POAF, including BNP level and other clinical factors, for use with patients undergoing anatomic lung or esophageal surgery.

\section{MATERIALS AND METHODS}

Following approval from the Memorial Sloan Kettering Cancer Center Institutional Review Board, we examined the charts of patients who underwent routine preoperative BNP measurement within 30 days before lung or esophageal resection $(n=1800)$ as part of our standard of care between April 2009 and March 2012. Excluded patients are described in the flow diagram (Figure 1). The final analysis set included 635 patients who underwent anatomic lung or esophageal resection. ${ }^{1,3,6}$ No additional BNP measurements were taken during the postoperative period. The current study cohort included a subset of patients $(n=269)$ from our previously published study on the association between BNP and POAF. ${ }^{11}$ Patients with a history of paroxysmal atrial fibrillation $(\mathrm{AF})$ but who were in sinus rhythm at the time of preoperative evaluation and in the operating room were included in the study. The BNP measurements were performed using an Alere Triage Meter (Alere North America, Orlando, Fla), which has a reportable range of 5 to $3200 \mathrm{pg} / \mathrm{mL}$. Following surgery, patients were transferred from the postanesthesia care unit to the thoracic surgical floor on postoperative day 1 . To avoid withdrawal symptoms, preoperative $\beta$-blockers and calcium channel blockers were continued; additional use of these medications for early postoperative management in other patients was recorded. Patients were monitored with continuous telemetry for 48 to 72 hours on average, or longer if needed. The primary clinical end point of the study was the new onset of POAF lasting $>5$ minutes during hospitalization and detected by continuous telemetry or 12-lead echocardiogram if the patient developed clinically significant POAF after telemetry was discontinued.

No formal power analysis was performed for this study. We focused on statistical power provided by the proposed prediction model on the basis of the reliability of predictions, rather than on specific predictors. The minimum events per variable to obtain good prediction is commonly set at 10 for logistic regression analysis. With an anticipated historical POAF rate of $20 \%$ and an anticipated selection of 6 final factors (5 degrees of freedom), the minimum required sample size must be at least 300 to meet the criterion of events per variable $\geq 10$. $^{13}$

Clinical characteristics were summarized by POAF status. The relationships between clinical factors and POAF were quantified using univariable logistic regression models. There were no missing data in this cohort. The

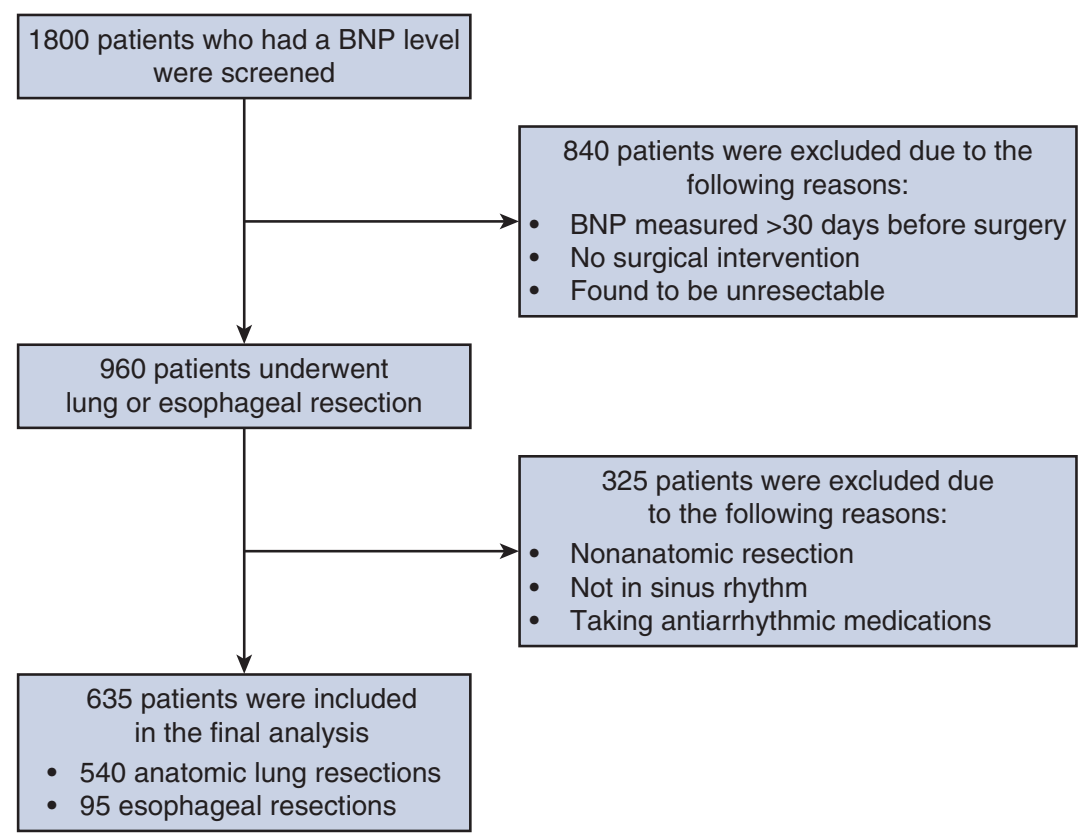

FIGURE 1. Study flow diagram showing patients excluded from the study and the final cohort included in the study analysis. $B N P$, Brain natriuretic peptide. 
linearity assumptions for continuous variables were examined using restricted cubic splines with at least 3 knots. Restricted cubic splines were fit using the rcspline.eval function in the rms R package (R Foundation for Statistical Computing, Vienna, Austria), with 3 knots placed at the 10th, 50th, and 90th percentile of the continuous variable. To develop the prediction model, a multivariable logistic regression model for the probability of POAF was constructed using a backward selection strategy, starting with factors with $P \leq .1$ in univariable analyses. The final prediction model was translated into a nomogram to provide an estimate of the probability of POAF following anatomic lung or esophageal resection using a visual point system. This study followed the TRIPOD Checklist: Prediction Model Development (see Online Data Supplement).

The performance of the prediction model was assessed by examining the discrimination ( $C$ index) and calibration (calibration plots). The $C$ index describes the predictive accuracy of the model, and values closer to 1 indicate superior discriminatory ability. Calibration plots were generated to compare the predicted probability of POAF from the prediction model with the observed occurrence of POAF, by dividing patients into groups (or bins) on the basis of predicted probability of POAF. An ideal prediction model would yield a plot where the observed and predicted probabilities perfectly correspond and align along the $45^{\circ}$ line. We also present the Hosmer-Lemeshow goodness-of-fit test to evaluate the model's fit. In addition, we present the net reclassification improvement index to measure the improvement with the addition of BNP to the prediction model.

To address overfitting and to quantify optimism, the prediction model was internally validated using a bootstrap-resampling approach. Optimism represents the difference between the apparent (unadjusted) performance measure and bootstrap (bias-) corrected measures. The optimism-corrected $C$ index and calibration curves were derived on the basis of 1000 bootstrap resamples of the original sample. All statistical tests were 2 -sided, and $P<.05$ indicates statistical significance. Statistical analyses were conducted with Stata version 13.1 (StataCorp, College Station, Tex) and $\mathrm{R}$ version 3.5.1 using the rms, pROC, predi$c A B E L$, and Hmisc packages.

\section{RESULTS}

Patient characteristics and surgical data for the study cohort of 635 patients are presented in Table 1. The outcome of interest, POAF, occurred in $20 \%$ of patients (124 out of 635) at a median of 2 days (range, 0-6 days) after surgery. Length of hospital stay was significantly longer among patients who developed POAF (median, 8 days; 25th-75th percentile, 5-12.5 days]) than among those who did not (median, 5 days; 25th-75th percentile, 4-8 days; $P<.0001)$. In univariable analyses, patients who developed POAF were older (median, 72 vs 68 years; $P<.0001$ ) and more likely to be men ( $65 \%$ vs $50 \% ; P=.003)$, have hypertension ( $66 \%$ vs $56 \% ; P=.043)$, and use preoperative $\beta$-blockers $(42 \%$ vs $26 \% ; P=.001)$, compared with patients who did not develop POAF. Patients with a history

TABLE 1. Demographic and clinical characteristics of patients by postoperative atrial fibrillation (POAF) status and univariable logistic regression results for the odds of $\mathrm{POAF}$

\begin{tabular}{|c|c|c|c|c|}
\hline \multirow[b]{2}{*}{ Characteristic } & \multirow[b]{2}{*}{ POAF $(n=124)(20 \%) *$} & \multirow[b]{2}{*}{ No POAF $(n=511)(80 \%) *$} & \multicolumn{2}{|c|}{ Univariable logistic regression } \\
\hline & & & OR $(95 \%$ confidence interval $)$ & $P$ value \\
\hline Age (y) & $71(67-77)$ & $68(63-74)$ & $1.05(1.03-1.08)$ & $<.0001$ \\
\hline Male & $81(65)$ & $256(50)$ & $1.88(1.25-2.82)$ & .003 \\
\hline Body mass index & $28(25-31)$ & $27(24-30)$ & $1.04(1.00-1.07)$ & .027 \\
\hline Smoking history & $101(81)$ & $411(80)$ & $1.07(0.65-1.77)$ & .8 \\
\hline Hypertension & $82(66)$ & $286(56)$ & $1.54(1.02-2.32)$ & .041 \\
\hline Coronary artery disease & $26(21)$ & $90(18)$ & $1.24(0.76-2.02)$ & .4 \\
\hline Chemotherapy & $45(36)$ & $169(33)$ & $1.15(0.77-1.74)$ & .5 \\
\hline Diabetes mellitus & $18(15)$ & $66(13)$ & $1.14(0.65-2.01)$ & 6 \\
\hline History of atrial fibrillation & $16(13)$ & $11(2.2)$ & $6.73(3.04-14.92)$ & $<.0001$ \\
\hline Preoperative $\beta$-blocker use & $52(42)$ & $134(26)$ & $2.03(1.35-3.05)$ & .001 \\
\hline Preoperative calcium blocker use & $9(7.3)$ & $21(4.1)$ & $1.83(0.81-4.09)$ & .14 \\
\hline Preoperative heart rate (bpm) & $70(63-80)$ & $72(64-81)$ & $1.00(0.98-1.02)$ & .9 \\
\hline $\mathrm{BNP}(\mathrm{pg} / \mathrm{mL}) \dagger$ & $45(20-94)$ & $23(11-50)$ & $2.64(1.68-4.16) \ddagger$ & $<.0001$ \\
\hline \multicolumn{5}{|l|}{ Extent of surgery } \\
\hline Segmentectomy & $7(5.6)$ & $57(11)$ & 1.0 & \\
\hline Lobectomy & $80(65)$ & $375(73)$ & $1.74(0.76-3.95)$ & .2 \\
\hline Pneumonectomy & $9(7.3)$ & $12(2.3)$ & $6.11(1.90-19.63)$ & .002 \\
\hline Esophagectomy & $28(23)$ & $67(13)$ & $3.40(1.38-8.37)$ & .008 \\
\hline Postoperative $\beta$-blocker use & $73(59)$ & $287(56)$ & $1.12(0.75-1.66)$ & 6 \\
\hline Postoperative calcium blocker use & $38(31)$ & $143(28)$ & $1.14(0.74-1.74)$ & .6 \\
\hline
\end{tabular}

Values are presented as n (\%) or median (25th-75th percentile), unless otherwise noted. $P O A F$, Postoperative atrial fibrillation; $B N P$, brain natriuretic peptide. $*$ Percentages in parentheses indicate the proportion of patients who have POAF or no POAF, respectively. $\dagger$ Odds ratio for brain natriuretic peptide is derived from 75 th percentile versus 25 th percentile $(57.5 \mathrm{vs} 12.5 \mathrm{pg} / \mathrm{mL}$ ) on the basis of restricted cubic splines with 3 knots. $\Varangle$ Nonlinear component in univariable model: $P=.004$. 
TABLE 2. Multivariable logistic regression model for the probability of postoperative atrial fibrillation

\begin{tabular}{lccc}
\hline \multicolumn{1}{c}{ Variable } & $\begin{array}{c}\text { Odds ratio }(95 \% \\
\text { Confidence interval) }\end{array}$ & $\begin{array}{c}\boldsymbol{P} \\
\text { value }\end{array}$ & $\begin{array}{c}\text { Overall } \\
\boldsymbol{P} \text { value }\end{array}$ \\
\hline Age $(\mathrm{y})$ & $1.05(1.02-1.08)$ & .001 & .001 \\
Body mass index & $1.05(1.00-1.09)$ & .016 & .016 \\
$\begin{array}{l}\text { Brain natriuretic peptide } \\
\quad \text { (pg/mL)* }\end{array}$ & $2.08(1.26-3.43)$ & .0003 & .0003 \\
$\quad$ History of atrial fibrillation & $5.91(2.47-14.11)$ & $<.0001$ & $<.0001$ \\
Operation type $\dagger$ & & & .0002 \\
$\quad$ Lobectomy & $1.88(0.83-4.90)$ & .16 & \\
$\quad \begin{array}{l}\text { Pneumonectomy } \\
\quad \text { Esophagectomy }\end{array}$ & $6.70(1.91-24.70)$ & .003 & \\
\hline
\end{tabular}

Apparent $C$ index, 736; optimism-corrected $C$ statistic, 0.720 (optimism-corrected value is based on 1000 bootstrap resamples). Hosmer-Lemeshow goodness-of-fit test: $P=.3$. *Odds ratio for brain natriuretic peptide is derived from 75 th percentile versus 25 th percentile $(57.5 \mathrm{vs} 12.5 \mathrm{pg} / \mathrm{mL}$ ) on the basis of restricted cubic splines with 3 knots. $\dagger$ Reference operation type: segmentectomy.

of $\mathrm{AF}$ were also more likely to experience $\mathrm{POAF}$, compared with those without a history of $\mathrm{AF}(13 \%$ vs $2 \% ; P<.0001)$. Esophagectomy and pneumonectomy were associated with a higher rate of POAF, compared with segmentectomy (overall Wald test, $P=.0002$ ). The incidence of POAF was similar between video-assisted thoracic surgery (VATS) and standard open thoracotomy for anatomic lung resection (38 out of $194[19.6 \%]$ vs 58 out of 346 $[16.7 \%] ; P=.41)$. The incidence of POAF did not differ significantly between minimally invasive surgery and standard open abdominal-thoracic esophagectomy (5 out of 12 [41.7\%] vs 23/83 [27.7\%]; $P=.32$ ). The use of metoprolol or diltiazem in the immediate postoperative period did not differ by POAF status (Table 1). The distribution of BNP level was right-skewed: only $10 \%$ of patients had BNP level $>100 \mathrm{pg} / \mathrm{mL}$, and $3 \%$ had BNP level $>200 \mathrm{pg} / \mathrm{mL}$. Across all patients, the median BNP level was $25 \mathrm{pg} / \mathrm{mL}$ (25th-75th percentile, $12-58 \mathrm{pg} / \mathrm{mL}$ ), and BNP level was higher among patients with than without POAF (median, $45 \mathrm{pg} / \mathrm{mL}$; 25th75th percentile, 20-94 vs median, $23 \mathrm{pg} / \mathrm{mL}$; 25th-75th percentile, $11-50 \mathrm{pg} / \mathrm{mL} ; P<.0001)$.

\section{Development of the Prediction Model}

Eight preoperative variables (ie, age, male gender, body mass index $[\mathrm{BMI}]$, hypertension, coronary artery disease, preoperative use of $\beta$-blockers, BNP level [with restricted cubic spline], and extent of surgery) with $P$ values $\leq .1$ in univariable analyses were considered as candidates for multivariable logistic regression analysis. The linearity assumption was not rejected for age $(P=.6)$ or BMI $(P=.4)$ but was significant for BNP level on a univariable basis $(P=.004)$. The final multivariable model included age, BMI, BNP level (with restricted cubic splines), history of AF, and extent of surgery (eg, segmentectomy, lobectomy, pneumonectomy, or esophagectomy) (Table 2). On the basis of the final prediction model, we generated a nomogram for predicting the probability of POAF (Figure 2). We also constructed an interactive calculator (Table E2) to estimate the risk of POAF.

\section{Model Performance and Internal Validation}

The $C$ index for the prediction model was $0.736(95 \%$ confidence interval [CI], 0.690-0.784), indicating good discrimination. The Hosmer-Lemeshow goodness-of-fit statistic was $P=.317$. Calibration curves (Figure 3) were

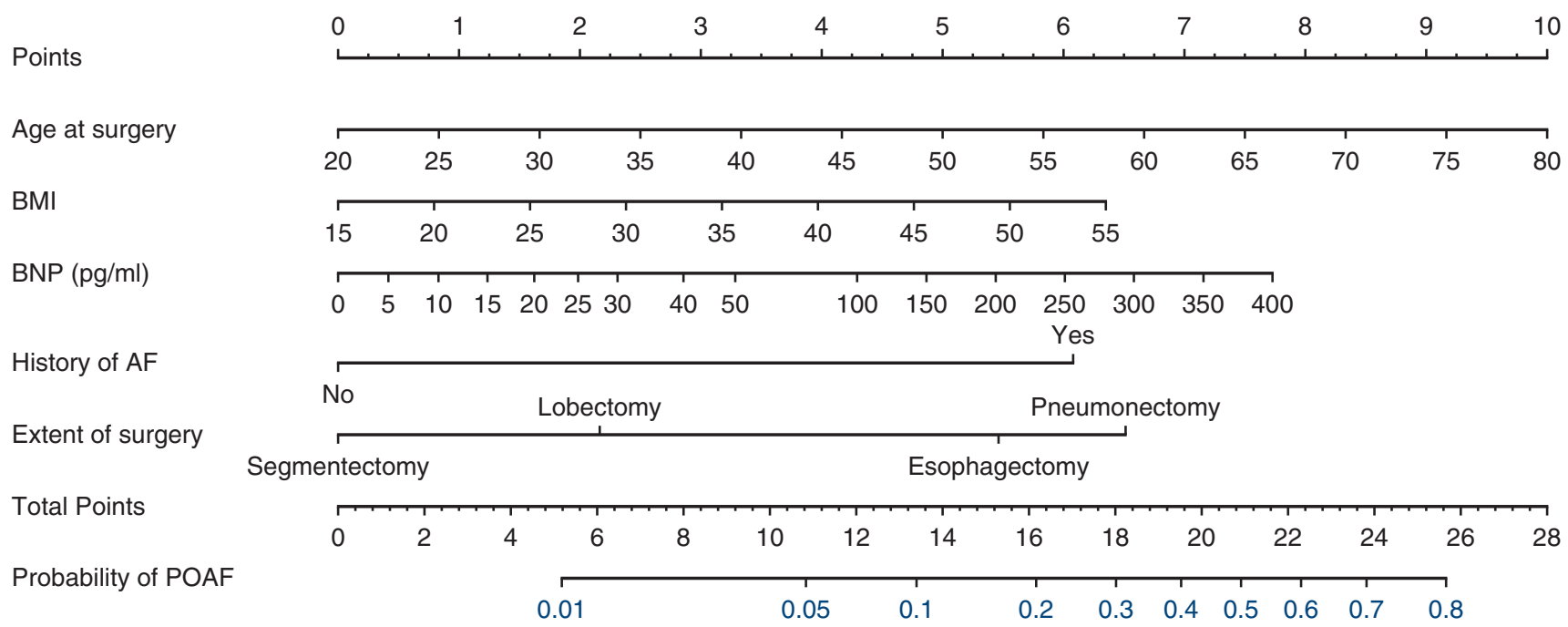

FIGURE 2. The nomogram presents a visual method to calculate the probability of postoperative atrial fibrillation $(P O A F)$ based on a patient's combination of characteristics. To calculate the probability of POAF, sum up the points identified on the scale for each of the five variables (age, body mass index [BMI], brain natriuretic peptide $[B N P]$ level, history of atrial fibrillation $[A F]$, and extent of surgery) to obtain the total points. Draw a vertical line down from the total points scale to the last axis to obtain the corresponding probability of POAF. 


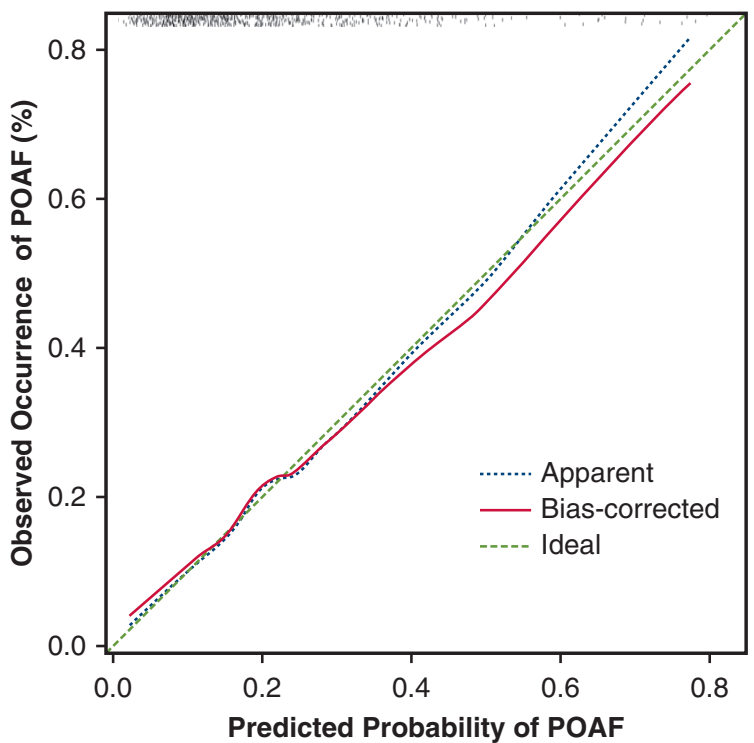

FIGURE 3. Calibration curves for the probability of postoperative atrial fibrillation $(P O A F)$ from the prediction model are shown. A perfectly accurate prediction model would yield a plot where the actual observed and predicted probabilities perfectly correspond and fall along the $45^{\circ}$ (dashed) line. The apparent calibration curve (dotted line) represents the model's calibration in the development data set, whereas the bias-corrected curve (solid line) is the calibration result after applying the bootstrapresampling procedure to correct for optimism (1000 bootstrapped resamples). POAF, Postoperative atrial fibrillation.

close to the $45^{\circ}$ ideal line, showing excellent agreement between the observed and predicted probabilities. Internal validation with the bootstrap approach was performed to address the optimism of the model. The optimismcorrected $C$ index of 0.720 (95\% CI, 0.664-0.765) reflected good discrimination, and the bias-corrected calibration curve similarly confirmed that the prediction model was well calibrated. To investigate the additive value of BNP level above clinical factors alone, we assessed the relative improvement in prediction with the addition of BNP level. The apparent $C$ index of the proposed model with BNP level was 0.737 (95\% CI, 0.689-0.782), which is higher than the $C$ index from the model excluding BNP level $(0.708 ; 95 \%$ CI, 0.657-0.753). This improvement in prediction with the inclusion of BNP level is reflected by net reclassification improvement (continuous) of 0.363 (95\% CI, 0.170 $0.556 ; P=.002)$ (Table E1).

\section{DISCUSSION}

On the basis of our previous examination of the association between BNP and POAF, we developed and internally validated a prediction model for the risk of POAF that included age, BMI, history of AF, extent of surgery, and preoperative BNP level. Using these risk factors, we developed a novel probability nomogram and an interactive calculator to estimate the likelihood of POAF occurrence, with good predictive ability for the model (Video 1). Figure 4 summarizes the study design, data, and development of the prediction model and interactive calculator. We showed that the model that included BNP level performed better than the model without BNP level. The overall incidence of POAF in our study was $20 \%$ (124 out of 635) and was associated with longer hospital stay. Although we included esophagectomy patients in our study, on the rationale that they have similar rates of POAF as patients undergoing anatomic lung resection, ${ }^{2}$ our multivariable model considered esophagectomy patients independently and showed that they have a greater risk of POAF, compared with patients undergoing anatomic lung resection, with the exception of pneumonectomy. The rate of POAF among esophagectomy patients was higher than the rate in an early series from our institution ${ }^{14}$ but was similar to the rate in more recently published work. ${ }^{15}$

Previously, examining a cohort of patients without available BNP data, we devised a clinical prediction rule and point score for POAF risk using the easily available preoperative clinical characteristics of age $>55$ years, male gender, and preoperative heart rate $>72 \mathrm{bpm}$, with age as the most important risk factor. ${ }^{1}$ In the current cohort, preoperative heart rate did not discriminate patients likely to develop POAF (Table 1), possibly because of the higher rate of $\beta$-blocker use among these patients $(29 \%$ vs $13.8 \%$ in the previous study ${ }^{1}$ ). A recent post hoc analysis of a large cohort of patients undergoing noncardiac surgery identified increasing age and thoracic surgery as predictors of POAF among patients undergoing vascular or abdominal surgery. ${ }^{16}$ In contrast to that study, we focused on thoracic surgery patients only and used a proven biomarker (ie, BNP) to identify patients at risk of POAF. ${ }^{9-12}$ In the current study, we built on previous work showing that preoperative subclinical elevation in BNP level is associated with a greater risk of POAF. ${ }^{9,10,12}$ When we

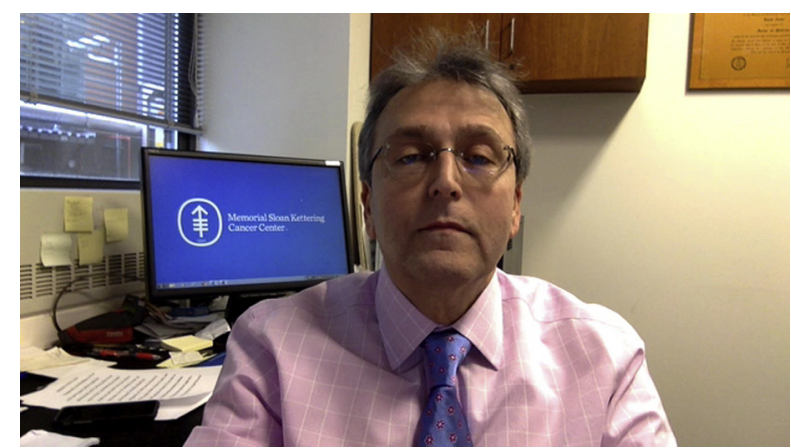

VIDEO 1. Dr Amar discusses the importance of the study findings to the practice of thoracic surgery. Video available at: https://www.jtcvs.org/ article/S0022-5223(19)30289-2/fulltext. 


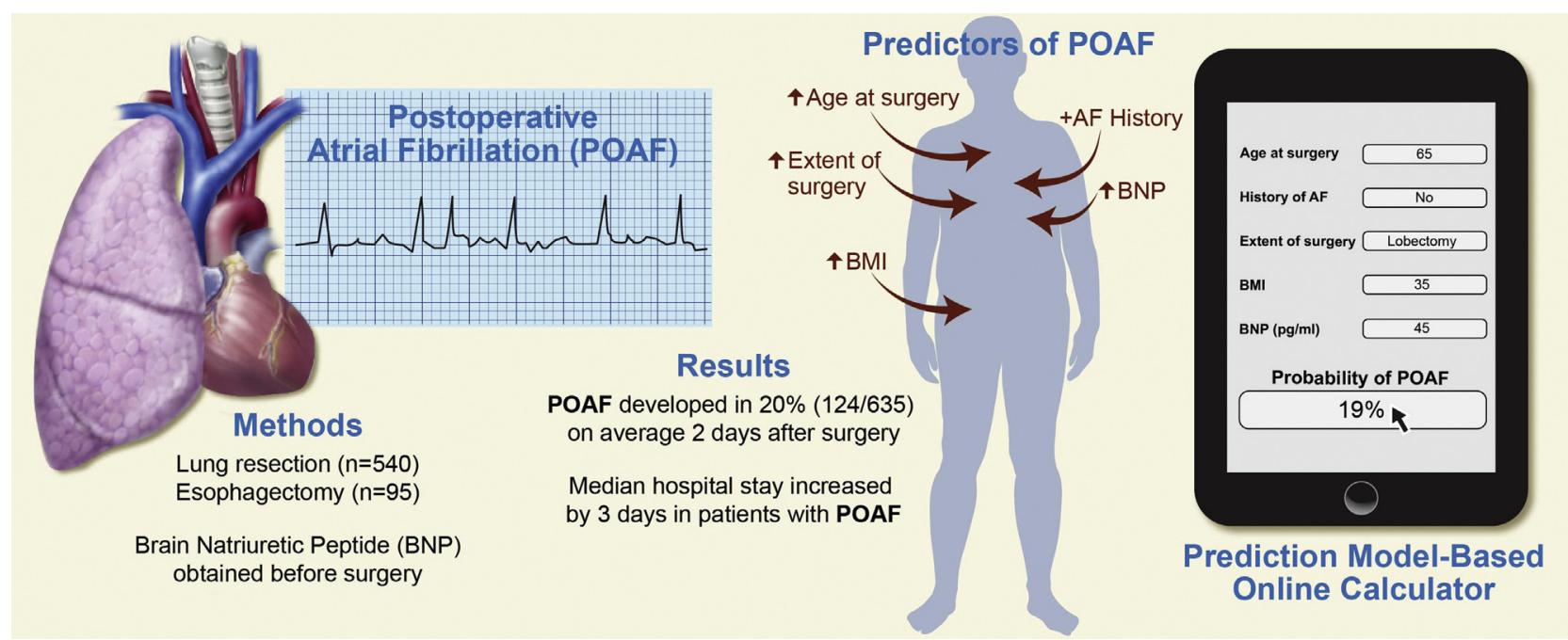

FIGURE 4. A pictorial summary of the incidence of postoperative atrial fibrillation $(P O A F)$ and its association with increased length of hospital stay. We present our model to calculate the risk of postoperative atrial fibrillation $(A F)$ using a novel brain natriuretic peptide $(B N P)$ based prediction rule. $B M I$, Body mass index.

directly compared BNP level to echocardiography-derived parameters to predict POAF in a similar population of patients, indices of left atrial and diastolic dysfunction, but not BNP level, were independently associated with greater risk of POAF. ${ }^{7}$ However, in that study, elevations in preoperative BNP level were correlated with older age and increased left atrial size and diastolic dysfunction among patients who mostly had no evidence of clinically significant cardiac dysfunction. ${ }^{7}$ Traditionally, elevated atrial natriuretic peptide levels have been demonstrated in patients with heart failure and either ventricular or atrial wall distension. BNP level may also increase with chronic conditions, such as aging, atrial fibrosis, and inflammation-factors that are also known to contribute to the occurrence of POAF in patients without clinical evidence of cardiac wall stretch or heart failure. ${ }^{17}$ In cases where an isolated abnormal BNP level is observed before surgery without clinical correlation, possible intervention to evaluate and optimize the patient's condition may be required. Taken together, these findings from the literature suggest that the presence of preoperative subclinical left atrial dysfunction (as measured by echocardiography or elevated BNP level) contributes to known postoperative aggravating conditions (such as systemic inflammation, oxidative stress, and altered sympathovagal balance) that play a role in initiating POAF in older patients undergoing major thoracic surgery. $2,4,18$

\section{Clinical Implications}

The clinical variables used in our prediction rule are quantifiable risk factors that are readily available to surgeons, anesthesiologists, or cardiologists before surgery. On the basis of these results, we have modified our practice to incorporate preoperative BNP measurement in the workup for patients scheduled for major resections, and eligible patients are considered for amiodarone prophylaxis, for example, as recommended by the American Association for Thoracic Surgery 2014 Taskforce. ${ }^{2}$ Others have used BNP cutoffs to guide small clinical trials to prevent POAF using novel medications, such as human atrial natriuretic peptide or olprinone, a phosphodiesterase III inhibitor, as well as more conventional drugs like $\beta$-blockers or angiotensin converting enzyme inhibitors. ${ }^{19-21}$

\section{Limitations}

Our patients were monitored with telemetry for 48 to 72 hours on average, or longer if necessary. However, our goal was to record clinically significant episodes of AF, which, in our experience as well as that of others, would consist of AF episodes that lasted $>5$ minutes to require intervention. It has also been our experience that approximately $10 \%$ of $\mathrm{AF}$ episodes are silent and may have occurred after telemetry was discontinued. We did not have accurate data on rates of readmission attributable to POAF following discharge from the hospital. In our experience, VATS lung resection carries a similar risk of POAF as open thoracotomy. ${ }^{22}$ We therefore included patients undergoing VATS procedures. The rate of POAF in the current study did not differ meaningfully between VATS and standard open thoracotomy for anatomic lung resection. Although no patients were excluded because of a history of heart failure or severe valvular heart disease, these are uncommon conditions in the population of patients referred to our institution for thoracic surgery. Our prediction model was derived from a single center, and because external validation data are not currently available, we performed 
internal validation by bootstrapping. Internal validation by bootstrapping has been shown to be superior to other internal validation methods (eg, split-sample) and is thus a recommended approach for predictive logistic regression models. ${ }^{14}$ However, we emphasize that no internal validation methods can substitute for external validation, and we stress that external validation is still necessary to determine whether our proposed prediction model can be generalized to an external population.

\section{CONCLUSIONS}

To our knowledge, the prediction rule described here, which uses preoperative BNP level together with age, BMI, extent of surgery, and history of AF to predict the risk of POAF after thoracic surgery, is a novel development. We propose that the novel prediction-model-based interactive calculator described here might be used to identify patients at high risk of POAF and could be incorporated into practice prevention guidelines. ${ }^{2,23}$

\section{Conflict of Interest Statement}

Authors have nothing to disclose with regard to commercial support.

The authors thank David Sewell, Department of Surgery, Memorial Sloan Kettering Cancer Center, for providing editorial assistance.

\section{References}

1. Passman RS, Gingold DS, Amar D, Lloyd-Jones D, Bennett CL, Zhang H, et al. Prediction rule for atrial fibrillation after major noncardiac thoracic surgery. Ann Thorac Surg. 2005;79:1698-703.

2. Frendl G, Sodickson AC, Chung MK, Waldo AL, Gersh BJ, Tisdale JE, et al. 2014 AATS guidelines for the prevention and management of perioperative atrial fibrillation and flutter for thoracic surgical procedures. Executive summary. $J$ Thorac Cardiovasc Surg. 2014;148:772-91.

3. Gialdini G, Nearing K, Bhave PD, Bonuccelli U, Iadecola C, Healey JS, et al. Perioperative atrial fibrillation and the long-term risk of ischemic stroke. JAMA. 2014;312:616-22.

4. Amar D, Zhang H, Miodownik S, Kadish AH. Competing autonomic mechanisms precede the onset of postoperative atrial fibrillation. J Am Coll Cardiol. 2003:42:1262-8.

5. Nojiri T, Maeda H, Takeuchi Y, Funakoshi Y, Maekura R, Yamamoto K, et al. Predictive value of preoperative tissue Doppler echocardiographic analysis for postoperative atrial fibrillation after pulmonary resection for lung cancer. $J$ Thorac Cardiovasc Surg. 2010;140:764-8.

6. Raman T, Roistacher N, Liu J, Zhang H, Shi W, Thaler HT, et al. Preoperative left atrial dysfunction and risk of postoperative atrial fibrillation complicating thoracic surgery. J Thorac Cardiovasc Surg. 2012;143:482-7.

7. Brecher O, Gulati H, Roistacher N, Zhang H, Shi W, Thaler HT, et al. Preoperative echocardiographic indices of diastolic dysfunction and brain natriuretic peptide in predicting postoperative atrial fibrillation after noncardiac surgery. Anesth Analg. 2017;124:1099-104.
8. Karthikeyan G, Moncur RA, Levine O, Heels-Ansdell D, Chan MT, AlonsoCoello $\mathrm{P}$, et al. Is a pre-operative brain natriuretic peptide or $\mathrm{N}$-terminal proB-type natriuretic peptide measurement an independent predictor of adverse cardiovascular outcomes within 30 days of noncardiac surgery? A systematic review and meta-analysis of observational studies. J Am Coll Cardiol. 2009; 54:1599-606.

9. Nojiri T, Inoue M, Shintani Y, Takeuchi Y, Maeda H, Hamasaki T, et al. B-type natriuretic peptide-guided risk assessment for postoperative complications in lung cancer surgery. World J Surg. 2015;39:1092-8.

10. Cardinale D, Colombo A, Sandri MT, Lamantia G, Colombo N, Civelli M, et al Increased perioperative $\mathrm{N}$-terminal pro-B-type natriuretic peptide levels predict atrial fibrillation after thoracic surgery for lung cancer. Circulation. 2007;115 1339-44.

11. Amar D, Zhang H, Shi W, Downey RJ, Bains MS, Park BJ, et al. Brain natriuretic peptide and risk of atrial fibrillation after thoracic surgery. J Thorac Cardiovasc Surg. 2012; 144:1249-53.

12. Pu Z, Qi X, Xue T, Liu Z, Wu Y. B-type natriuretic peptide and other risk factors for predicting postoperative atrial fibrillation after thoracic surgery. Thorac Cardiovasc Surg. 2019;67:120-4.

13. Steyerberg EW, Harrell FE Jr, Borsboom GJ, Eijkemans MJ, Vergouwe Y, Habbema JD. Internal validation of predictive models: efficiency of some procedures for logistic regression analysis. J Clin Epidemiol. 2001;54:774-81.

14. Amar D, Burt ME, Bains MS, Leung DH. Symptomatic tachydysrhythmias after esophagectomy: incidence and outcome measures. Ann Thorac Surg. 1996;61: 1536-9.

15. Mc Cormack O, Zaborowski A, King S, Healy L, Daly C, O’Farrell N, et al. Newonset atrial fibrillation post-surgery for esophageal and junctional cancer incidence, management, and impact on short- and long-term outcomes. Ann Surg. 2014;260:772-8.

16. Alonso-Coello P, Cook D, Xu SC, Sigamani A, Berwanger O, Sivakumaran S, et al. Predictors, prognosis, and management of new clinically important atrial fibrillation after noncardiac surgery: a prospective cohort study. Anesth Analg. 2017;125:162-9.

17. Marsiliani D, Buccelletti F, Carroccia A, Gilardi E, Silveri NG, Franceschi F. Natriuretic peptides and atrial fibrillation. Eur Rev Med Pharmacol Sci. 2010; 14:855-60.

18. January CT, Wann LS, Alpert JS, Calkins H, Cigarroa JE, Cleveland JC Jr, et al 2014 AHA/ACC/HRS guideline for the management of patients with atrial fibrillation: a report of the American College of Cardiology/American Heart Association task force on practice guidelines and the Heart Rhythm Society. J Am Coll Cardiol. 2014;64:e1-76.

19. Nojiri T, Yamamoto K, Maeda H, Takeuchi Y, Funakoshi Y, Inoue M, et al. Effect of low-dose human atrial natriuretic peptide on postoperative atrial fibrillation in patients undergoing pulmonary resection for lung cancer: a double-blind, placebo-controlled study. J Thorac Cardiovasc Surg. 2012;143:488-94.

20. Nojiri T, Yamamoto K, Maeda H, Takeuchi Y, Ose N, Susaki Y, et al. A doubleblind placebo-controlled study of the effects of olprinone, a specific phosphodiesterase III inhibitor, for preventing postoperative atrial fibrillation in patients undergoing pulmonary resection for lung cancer. Chest. 2015;148:1285-92.

21. Cardinale D, Sandri MT, Colombo A, Salvatici M, Tedeschi I, Bacchiani G, et al Prevention of atrial fibrillation in high-risk patients undergoing lung cancer surgery: the PRESAGE trial. Ann Surg. 2016;264:244-51.

22. Park BJ, Zhang H, Rusch VW, Amar D. Video-assisted thoracic surgery does not reduce the incidence of postoperative atrial fibrillation after pulmonary lobectomy. J Thorac Cardiovasc Surg. 2007;133:775-9.

23. Amar D. Postoperative atrial fibrillation: is there a need for prevention? J Thorac Cardiovasc Surg. 2016;151:913-5.

Key Words: arrhythmias, complications, lung resection, esophagectomy 
TABLE E1. Reclassification table based on the full model with brain natriuretic peptide (BNP) level versus the full model without BNP level

\begin{tabular}{|c|c|c|c|c|}
\hline \multirow{3}{*}{$\begin{array}{l}\text { Full model without } \\
\text { BNP, Prob }\end{array}$} & \multicolumn{3}{|c|}{ Full model with BNP, Prob } & \multirow[b]{3}{*}{$\%$ reclassified } \\
\hline & & $15 \%$ to & & \\
\hline & $<\mathbf{1 5} \%$ & $<\mathbf{3 0} \%$ & $\geq \mathbf{3 0} \%$ & \\
\hline \multicolumn{5}{|l|}{ No POAF } \\
\hline$<15 \%$ & 226 & 30 & 2 & 12 \\
\hline $15 \%$ to $<30 \%$ & 60 & 105 & 28 & 46 \\
\hline$\geq 30 \%$ & 0 & 19 & 41 & 32 \\
\hline \multicolumn{5}{|l|}{ POAF } \\
\hline$<15 \%$ & 22 & 8 & 1 & 29 \\
\hline $15 \%$ to $<30 \%$ & 7 & 31 & 12 & 38 \\
\hline$\geq 30 \%$ & 0 & 4 & 39 & 9 \\
\hline \multicolumn{5}{|l|}{ Combined } \\
\hline$<15 \%$ & 248 & 38 & 3 & 14 \\
\hline $15 \%$ to $<30 \%$ & 67 & 136 & 40 & 44 \\
\hline$\geq 30 \%$ & 0 & 23 & 80 & 22 \\
\hline
\end{tabular}

Variables in the full model include age, body mass index, history of atrial fibrillation, and extent of surgery. Categorical net reclassification index, $0.118(95 \%$ confidence interval, 0.019-0.217; $P=.020)$. Continuous net reclassification index, $0.390(95 \%$ confidence interval, $0.196-0.583 ; P<.0001) . B N P$, Brain natriuretic peptide; Prob $_{P O A}$, predicted probability of POAF; $P O A F$, postoperative atrial fibrillation.

TABLE E2. Interactive calculator to estimate the probability of postoperative atrial fibrillation (POAF) given user input of age, body mass index (BMI), brain natriuretic peptide (BNP) level, history of atrial fibrillation (AF), and extent of surgery

\begin{tabular}{llcccc}
\hline & & \multicolumn{3}{c}{ Examples } \\
\cline { 3 - 5 } & & Patient 1 & Patient 2 & Patient 3 & Patient 4 \\
\hline Age (y) & $75^{*}$ & 50 & 50 & 50 & 75 \\
BMI & $35^{*}$ & 35 & 35 & 35 & 85 \\
BNP (0 to 450) & $200^{*}$ & 25 & 25 & No & No \\
History of AF & No* & Yes & Esophagectomy & Segmentectomy & Lobectomy \\
Extent of surgery & Lobectomy* & Esophagectomy & 17.60 & 32.20 & 43.00 \\
Probability of post-operative AF (\%) & $43 \dagger$ & 55.70 & & & \\
$95 \%$ Confidence interval (\%) & $(28.9-58.3)$ & & &
\end{tabular}

"Mr X, if we had 100 persons exactly like you (same BNP, age, BMI, history of AF, and extent of surgery), we would expect ___ \% patients to experience postoperative AF. The $95 \%$ confidence interval for this $\%$ is between_ and _ $\%$." *Patient's characteristics. †Patient's probability of postoperative AF. 